55, 4, pp. 1437-1441, Warsaw 2017

DOI: $10.15632 /$ jtam-pl.55.4.1437

\title{
JERK BY AXES IN MOTION ALONG A SPACE CURVE
}

\author{
Michael TSIRLIN \\ ELMO Motion Control, Petah Tiqva, Israel \\ e-mail:mz695444@gmail.com
}

\begin{abstract}
In the paper, a formula for calculation of the jerk of a point moving along a space curve is derived. Such a formula is needed for control of motion to calculate jerks (second derivatives of the velocity) by axes of motion. On the basis of this formula, an expression for the maximum admissible velocity on the space curve to satisfy limitation by the jerk is given as well. Such a requirement also arises in the motion control.
\end{abstract}

Keywords: jerk, motion control, profiler

\section{Introduction}

In the multi axis motion control, a motion command may contain four kinematics parameters: position, velocity, acceleration and jerk. Such a command must be issued for each axis $x, y$ and $z$ controlled by a servo on each control cycle. While calculation of the velocity and acceleration is trivial, calculation of jerk (that is mainly used for analysis of motion) is a new demand. In a number of papers devoted to the motion control, a tangential jerk along the trajectory is considered while the problem of jerk caused by a high curvature on the transition curve between two line segments is a new one. For a particular case of planar motion, the jerk vector was considered by Shot (1978). In this special case, the jerk can be resolved into tangential and normal components. As will be shown below, in the case of 3D space motion, the jerk vector has three components although the acceleration vector still can be decomposed into tangential and normal.

Consider a space curve in the 3D space defined by a vector function $\gamma(\sigma)=$ $\left\{\varphi_{x}(\sigma), \varphi_{y}(\sigma), \varphi_{z}(\sigma)\right\}$. In further considerations, any special nature of the parameter $\sigma$ ( $\sigma$ - is not time or arc length $s$ ) and any special limit of the parameter variation are not supposed. We suppose here that $\gamma(\sigma)$ is a continuous mapping $\sigma \rightarrow R_{3}, \sigma \in[a, b]$ with three times differentiable coordinate functions $\varphi_{x}(\sigma), \varphi_{y}(\sigma), \varphi_{z}(\sigma)$ that define geometrical properties of the curve.

As $\gamma^{\prime}(\sigma)=\left\{\varphi_{x}^{\prime}(\sigma), \varphi_{y}^{\prime}(\sigma), \varphi_{z}^{\prime}(\sigma)\right\}$ then the unit tangent vector can be defined as $\boldsymbol{\tau}(\sigma)=$ $\left\{\varphi_{x}^{\prime}(\sigma) /\left\|\boldsymbol{\gamma}^{\prime}(\sigma)\right\|, \varphi_{y}^{\prime}(\sigma) /\left\|\boldsymbol{\gamma}^{\prime}(\sigma)\right\|, \varphi_{z}^{\prime}(\sigma) /\left\|\boldsymbol{\gamma}^{\prime}(\sigma)\right\|\right\}$.

The second derivative $\mathbf{a}(\sigma)=\gamma^{\prime \prime}(\sigma)=\left\{\varphi_{x}^{\prime \prime}(\sigma), \varphi_{y}^{\prime \prime}(\sigma), \varphi_{z}^{\prime \prime}(\sigma)\right\}$ with a normal component $\mathbf{a}_{n}(\sigma)=\mathbf{a}(\sigma)-[\mathbf{a}(\sigma) \cdot \boldsymbol{\tau}(\sigma)] \boldsymbol{\tau}(\sigma)$. Then, the unit normal vector can be defined as $\mathbf{n}=\mathbf{a}_{n}(\sigma) /\left\|\mathbf{a}_{n}(\sigma)\right\|$.

The binormal unit vector $\mathbf{b}(\sigma)$ can be defined as $\boldsymbol{\tau}(\sigma) \times \mathbf{n}(\sigma)$. Thus, we know the Frenet-Serret frame $\boldsymbol{\tau}(\sigma), \mathbf{n}(\sigma)$ and $\mathbf{b}(\sigma)$ for each parameter value $\sigma$.

Consider a point moving along a curve defined by its radius-vector $\mathbf{r}(t)$. For each control cycle (at time $t$ ) from the profiler calculations we know the position increment along the curve $s(t)$, velocity along the curve $d s / d t=v(t)$, acceleration along the curve $d^{2} s / d t^{2}=a(t)$ and jerk along the curve $d^{3} s / d t^{3}=J(t)$, where $s$ is a distance along the curve. The value of the curve parameter $\sigma$ is not known but with the use of numerical methods, the mapping $s \rightarrow \sigma$ can be done with any required precision. 


\section{Vector of jerks for the position increment $s(t)$}

The velocity and acceleration vectors of the point moving along the space curve are defined by

$$
\begin{aligned}
& \mathbf{v}(t)=\frac{d \mathbf{r}}{d t}=\boldsymbol{\tau}\left(\frac{d s}{d t}\right) \\
& \mathbf{a}(t)=\frac{d \mathbf{v}}{d t}=\boldsymbol{\tau}\left(\frac{d^{2} s}{d t^{2}}\right)+\mathbf{n}\left(\frac{d s}{d t}\right)^{2} \frac{1}{\rho(s)}=\boldsymbol{\tau}\left(\frac{d^{2} s}{d t^{2}}\right)+\mathbf{n}\left(\frac{d s}{d t}\right)^{2} K_{1}(s)
\end{aligned}
$$

where $K_{1}(s)$ and $\rho(s)$ are the curvature and radius of the curvature at point $s$.

The vector of the third derivative (jerk) can be calculated as $d \mathbf{a}(t) / d t$

$$
\mathbf{J}(t)=\frac{d \mathbf{a}}{d t}=\frac{d \boldsymbol{\tau}}{d s} \frac{d s}{d t} \frac{d^{2} s}{d t^{2}}+\boldsymbol{\tau}\left(\frac{d^{3} s}{d t^{3}}\right)+\frac{d \mathbf{n}}{d s}\left(\frac{d s}{d t}\right)^{3} K_{1}(s)+\mathbf{n} \frac{d\left[\left(\frac{d s}{d t}\right)^{2} K_{1}(s)\right]}{d t}
$$

At first, we consider

$$
\mathbf{n} \frac{d\left[\left(\frac{d s}{d t}\right)^{2} K_{1}(s)\right]}{d t}=\mathbf{n}\left[2 \frac{d s}{d t} \frac{d^{2} s}{d t^{2}} K_{1}(s)+\left(\frac{d s}{d t}\right)^{2} \frac{d K_{1}(s)}{d s} \frac{d s}{d t}\right]
$$

By the Frenet-Serret formulas

$$
\frac{d \boldsymbol{\tau}}{d s}=K_{1} \mathbf{n} \quad \frac{d \mathbf{n}}{d s}=-K_{1} \boldsymbol{\tau}+K_{2} \mathbf{b}
$$

The curvature $K_{1}$ and torsion $K_{2}$ for an arbitrary curve parameter $\sigma$ (which is not necessarily the arc length $s$ ) at the parameter point $\sigma$ are calculated by

$$
\begin{aligned}
& K_{1}(\sigma)=\frac{\left\|\gamma^{\prime}(\sigma) \times \gamma^{\prime \prime}(\sigma)\right\|}{\left\|\gamma^{\prime}(\sigma)\right\|^{3}} \\
& K_{2}(\sigma)=\frac{\left[\gamma^{\prime}(\sigma), \gamma^{\prime \prime}(\sigma), \gamma^{\prime \prime \prime}(\sigma)\right]}{\left\|\gamma^{\prime}(\sigma) \times \gamma^{\prime \prime}(\sigma)\right\|^{2}}=\frac{\left[\gamma^{\prime}(\sigma) \times \gamma^{\prime \prime}(\sigma)\right] \cdot \gamma^{\prime \prime \prime}(\sigma)}{\left\|\gamma^{\prime}(\sigma) \times \gamma^{\prime \prime}(\sigma)\right\|^{2}}
\end{aligned}
$$

where $\gamma^{\prime}(\sigma), \gamma^{\prime \prime}(\sigma), \gamma^{\prime \prime \prime}(\sigma)$ are known curve derivatives, and the curve parameter $\sigma$ can be found from the numerical mapping $s \rightarrow \sigma$ (if $\sigma \neq s$ ) that must be done for each point $s$. We calculate curvature and torsion at the parameter point $\sigma$ that corresponds to the increment $s$ along the curve. Point $s$ is a point where the profiler calculations have just determined $d s / d t, d^{2} s / d t^{2}$, $d^{3} s / d t^{3}$. So, at the point $s$, we can use $K_{1}(\sigma)$ and $K_{2}(\sigma)$ instead of $K_{1}(s)$ and $K_{2}(s)$. Below we use designations $K_{1}$ and $K_{2}$.

From (2.2)-(2.4), we get

$$
\begin{aligned}
\mathbf{J}(t) & =K_{1} \mathbf{n} \frac{d s}{d t} \frac{d^{2} s}{d t^{2}}+\boldsymbol{\tau}\left(\frac{d^{3} s}{d t^{3}}\right)+\left(-K_{1} \boldsymbol{\tau}+K_{2} \mathbf{b}\right)\left(\frac{d s}{d t}\right)^{3} K_{1} \\
& +\mathbf{n}\left[2 \frac{d s}{d t} \frac{d^{2} s}{d t^{2}} K_{1}+\left(\frac{d s}{d t}\right)^{2} \frac{d K_{1}(s)}{d s} \frac{d s}{d t}\right]=\boldsymbol{\tau}\left[\frac{d^{3} s}{d t^{3}}-K_{1}^{2}\left(\frac{d s}{d t}\right)^{3}\right] \\
& +\mathbf{n}\left[K_{1}(s) \frac{d s}{d t} \frac{d^{2} s}{d t^{2}}+2 \frac{d s}{d t} \frac{d^{2} s}{d t^{2}} K_{1}+\left(\frac{d s}{d t}\right)^{2} \frac{d K_{1}(s)}{d s} \frac{d s}{d t}\right]+\mathbf{b} \frac{K_{2}}{\rho}\left(\frac{d s}{d t}\right)^{3} \\
& =\boldsymbol{\tau}\left[\frac{d^{3} s}{d t^{3}}-\left(\frac{d s}{d t}\right)^{3} K_{1}^{2}\right]+\mathbf{n}\left[3 \frac{d s}{d t} \frac{d^{2} s}{d t^{2}} K_{1}+\left(\frac{d s}{d t}\right)^{3} \frac{d K_{1}(s)}{d s}\right]+\mathbf{b}\left[K_{2}\left(\frac{d s}{d t}\right)^{3} K_{1}\right]
\end{aligned}
$$

Since we do not know the function $K_{1}(s)$ then calculation of its derivative at the point $s$ requires special consideration. $K_{1}(s)=K_{1}[s(\sigma)]=K_{1 \sigma}(\sigma)$, and by differentiating both sides we get

$$
\frac{d K_{1}(s)}{d s}=\frac{d K_{1 \sigma}(\sigma)}{d s}=\frac{d K_{1 \sigma}(\sigma)}{d \sigma} \frac{d \sigma}{d s}=\frac{d K_{1 \sigma}(\sigma)}{d \sigma} / \frac{d s}{d \sigma}=\frac{d K_{1 \sigma}(\sigma)}{d \sigma} \frac{1}{\left\|\boldsymbol{\gamma}^{\prime}(\sigma)\right\|}
$$


where instead of the unknown $d \sigma / d s$ we used the derivative $d s / d \sigma=\left\|\gamma^{\prime}(\sigma)\right\|$ of the inverse function $s(\sigma)$, that is a curve length function

$$
\begin{aligned}
& \frac{d K_{1 \sigma}(\sigma)}{d \sigma}=\frac{d \frac{\left\|\boldsymbol{\gamma}^{\prime}(\sigma) \times \boldsymbol{\gamma}^{\prime \prime}(\sigma)\right\|}{\left\|\boldsymbol{\gamma}^{\prime}(\sigma)\right\|^{3}}}{d \sigma} \\
& \quad=\left(\frac{d\left\|\boldsymbol{\gamma}^{\prime}(\sigma) \times \gamma^{\prime \prime}(\sigma)\right\|}{d \sigma}\left\|\boldsymbol{\gamma}^{\prime}(\sigma)\right\|^{3}-\left\|\boldsymbol{\gamma}^{\prime}(\sigma) \times \boldsymbol{\gamma}^{\prime \prime}(\sigma)\right\| \frac{d\left\|\boldsymbol{\gamma}^{\prime}(\sigma)\right\|^{3}}{d \sigma}\right) \frac{1}{\left\|\boldsymbol{\gamma}^{\prime}(\sigma)\right\|^{6}} \\
& \quad=\frac{d\left\|\boldsymbol{\gamma}^{\prime}(\sigma) \times \boldsymbol{\gamma}^{\prime \prime}(\sigma)\right\|}{d \sigma} \frac{1}{\left\|\boldsymbol{\gamma}^{\prime}(\sigma)\right\|^{3}}-K_{1}(\sigma) \frac{d\left\|\boldsymbol{\gamma}^{\prime}(\sigma)\right\|^{3}}{d \sigma} \frac{1}{\left\|\boldsymbol{\gamma}^{\prime}(\sigma)\right\|^{3}}
\end{aligned}
$$

Below we assume that for any vector $\mathbf{p}$ the derivative of its Euclidean norm can be calculated as

$$
\begin{aligned}
& \|\mathbf{p}\|^{\prime}=\frac{1}{2}\left(\mathbf{p}^{\prime} \mathbf{p}+\mathbf{p} \mathbf{p}^{\prime}\right) \frac{1}{\sqrt{\mathbf{p p}}}=\frac{\mathbf{p} \mathbf{p}^{\prime}}{\|\mathbf{p}\|} \\
& \frac{d\left\|\gamma^{\prime}(\sigma) \times \gamma^{\prime \prime}(\sigma)\right\|}{d \sigma}=\frac{\left[\gamma^{\prime}(\sigma) \times \gamma^{\prime \prime}(\sigma)\right]\left[\gamma^{\prime \prime}(\sigma) \times \gamma^{\prime \prime}(\sigma)+\gamma^{\prime}(\sigma) \times \gamma^{\prime \prime \prime}(\sigma)\right]}{\left\|\gamma^{\prime}(\sigma) \times \gamma^{\prime \prime}(\sigma)\right\|} \\
& \quad=\frac{\left[\gamma^{\prime}(\sigma) \times \gamma^{\prime \prime}(\sigma)\right]\left[\gamma^{\prime}(\sigma) \times \gamma^{\prime \prime \prime}(\sigma)\right]}{\left\|\gamma^{\prime}(\sigma) \times \gamma^{\prime \prime}(\sigma)\right\|} \\
& \frac{d\left\|\gamma^{\prime}(\sigma)\right\|^{3}}{d \sigma}=3\left\|\gamma^{\prime}(\sigma)\right\|^{2} \frac{d\left\|\gamma^{\prime}(\sigma)\right\|}{d \sigma}=3\left\|\gamma^{\prime}(\sigma)\right\|^{2} \frac{\gamma^{\prime}(\sigma) \gamma^{\prime \prime}(\sigma)}{\left\|\gamma^{\prime}(\sigma)\right\|}=3\left\|\gamma^{\prime}(\sigma)\right\|\left[\gamma^{\prime}(\sigma) \boldsymbol{\gamma}^{\prime \prime}(\sigma)\right]
\end{aligned}
$$

So, finally we get

$$
\frac{d K_{1 \sigma}(\sigma)}{d \sigma}=\frac{\left[\gamma^{\prime}(\sigma) \times \gamma^{\prime \prime}(\sigma)\right]\left[\gamma^{\prime}(\sigma) \times \gamma^{\prime \prime \prime}(\sigma)\right]}{\left\|\gamma^{\prime}(\sigma)\right\|^{3}\left\|\gamma^{\prime}(\sigma) \times \gamma^{\prime \prime}(\sigma)\right\|}-3 K_{1}(\sigma) \frac{\gamma^{\prime}(\sigma) \gamma^{\prime \prime}(\sigma)}{\left\|\gamma^{\prime}(\sigma)\right\|^{2}}
$$

Another derivation of (2.10) was given by Angeles (2003, pp. 371-372).

In some cases (cubic spline with iterative adaptation of the parameter $\sigma$ or PH-curve proposed by Farouki (2010)) we can use the curve parameter close or equal to natural $\sigma=s$. In such cases, the curvature $K_{1}$ and its derivative $d K_{1} / d s$ are defined as

$$
\begin{aligned}
& K_{1}=\left\|\boldsymbol{\gamma}^{\prime \prime}(s)\right\|=\sqrt{\left[\varphi_{x}^{\prime \prime}(\sigma)\right]^{2}+\left[\varphi_{y}^{\prime \prime}(\sigma)\right]^{2}+\left[\varphi_{z}^{\prime \prime}(\sigma)\right]^{2}} \\
& \frac{d K_{1}}{d s}=\frac{1}{K_{1}}\left[\varphi_{x}^{\prime \prime}(\sigma) \varphi_{x}^{\prime \prime \prime}(\sigma)+\varphi_{y}^{\prime \prime}(\sigma) \varphi_{y}^{\prime \prime \prime}(\sigma)+\varphi_{z}^{\prime \prime}(\sigma) \varphi_{z}^{\prime \prime \prime}(\sigma)\right]=\frac{\boldsymbol{\gamma}^{\prime \prime}(\sigma) \boldsymbol{\gamma}^{\prime \prime \prime}(\sigma)}{\left\|\boldsymbol{\gamma}^{\prime \prime}(s)\right\|}
\end{aligned}
$$

In (2.6), we have come to the vector

$$
\mathbf{J}(t)=\boldsymbol{\tau}(\sigma) C_{1}+\mathbf{n}(\sigma) C_{2}+\mathbf{b}(\sigma) C_{3}
$$

The vectors $\boldsymbol{\tau}(\sigma), \mathbf{n}(\sigma)$ and $\mathbf{b}(\sigma)$ are defined above, and the scalar coefficients $C_{1}, C_{2}, C_{3}$ are also known from $(2.6)$

$$
\begin{aligned}
& C_{1}=\frac{d^{3} s}{d t^{3}}-\frac{K_{1}}{\rho}\left(\frac{d s}{d t}\right)^{3}=J(t)-K_{1}^{2} v^{3}(t) \\
& C_{2}=3 \frac{d s}{d t} \frac{d^{2} s}{d t^{2}} K_{1}+\left(\frac{d s}{d t}\right)^{3} \frac{d K_{1}(s)}{d s}=3 v(t) a(t) K_{1}+v^{3}(t) \frac{d K_{1}(\sigma)}{d \sigma} \frac{1}{\left\|\gamma^{\prime}(\sigma)\right\|} \\
& C_{3}=\frac{K_{2}}{\rho}\left(\frac{d s}{d t}\right)^{3}=K_{1} K_{2} v^{3}(t)
\end{aligned}
$$




\section{Applications of the formula}

In the case of uniform motion with $d s / d t=V, d^{2} s / d t^{2}=0$ and $d^{3} s / d t^{3}=0$ we get

$$
\begin{array}{ll}
C_{1}=-\frac{K_{1}}{\rho}\left(\frac{d s}{d t}\right)^{3}=-K_{1}^{2} V^{3} \quad C_{2}=\left(\frac{d s}{d t}\right)^{3} \frac{d K_{1}(s)}{d s}=V^{3} \frac{d K_{1}(\sigma)}{d \sigma} \frac{1}{\left\|\gamma^{\prime}(\sigma)\right\|} \\
C_{3}=\frac{K_{2}}{\rho}\left(\frac{d s}{d t}\right)^{3}=K_{1} K_{2} V^{3}
\end{array}
$$

In the case of plane $2 \mathrm{D}$ motion with $K_{2}=0$

$$
\begin{array}{ll}
C_{1} & =\frac{d^{3} s}{d t^{3}}-\frac{K_{1}}{\rho}\left(\frac{d s}{d t}\right)^{3}=J(t)-K_{1}^{2} v^{3}(t) \\
C_{2} & =3 v(t) a(t) K_{1}+v^{3}(t) \frac{d K_{1}(\sigma)}{d \sigma} \frac{1}{\left\|\boldsymbol{\gamma}^{\prime}(\sigma)\right\|} \quad C_{3}=0
\end{array}
$$

Formulas (3.1) can be used for the estimation of the maximum velocity $V$ admissible on the curve to satisfy condition $\|\mathbf{J}\| \leqslant J_{\max }$. While generating the trajectory, instead of $K_{1}(s)$ and $K_{2}(s)$ we know $K_{1}(\sigma)$ and $K_{2}(\sigma)$ for any parameter value $\sigma$. So, in the case of uniform motion with $d s / d t=V$ and designation $\widetilde{K}_{1}=\left[d K_{1}(\sigma) / d \sigma\right] /\left\|\gamma^{\prime}(\sigma)\right\|$ we get

$$
\begin{aligned}
& \|\mathbf{J}(\sigma)\|=\sqrt{K_{1}^{4}(\sigma) V^{6}+V^{6} \widetilde{K}_{1}^{2}+K_{1}^{2}(\sigma) K_{2}^{2}(\sigma) V^{6}} \\
& \quad=V^{3} \sqrt{K_{1}^{4}(\sigma)+\widetilde{K}_{1}^{2}+K_{1}^{2}(\sigma) K_{2}^{2}(\sigma)}=V^{3} \sqrt{\Psi(\sigma)}
\end{aligned}
$$

and the maximum velocity admissible at all trajectory points must satisfy

$$
V \leqslant \frac{\sqrt[3]{J_{\max }}}{\sqrt[6]{\max \Psi(\sigma)}}
$$

In most applications, the point $\sigma^{*}$ that produces $\Psi\left(\sigma^{*}\right)=\max \Psi(\sigma)$ belongs to a small neighborhood of the point $\sigma_{p}$ such that $\gamma\left(\sigma_{p}\right)$ can be called the peak of the curve.

In the case of $2 \mathrm{D}$ uniform motion, $d s / d t=V, K_{2}(\sigma)=0$, and we get

$$
\|\mathbf{J}(\sigma)\|=V^{3} \sqrt{K_{1}^{4}(\sigma)+\widetilde{K_{1}^{2}}}
$$
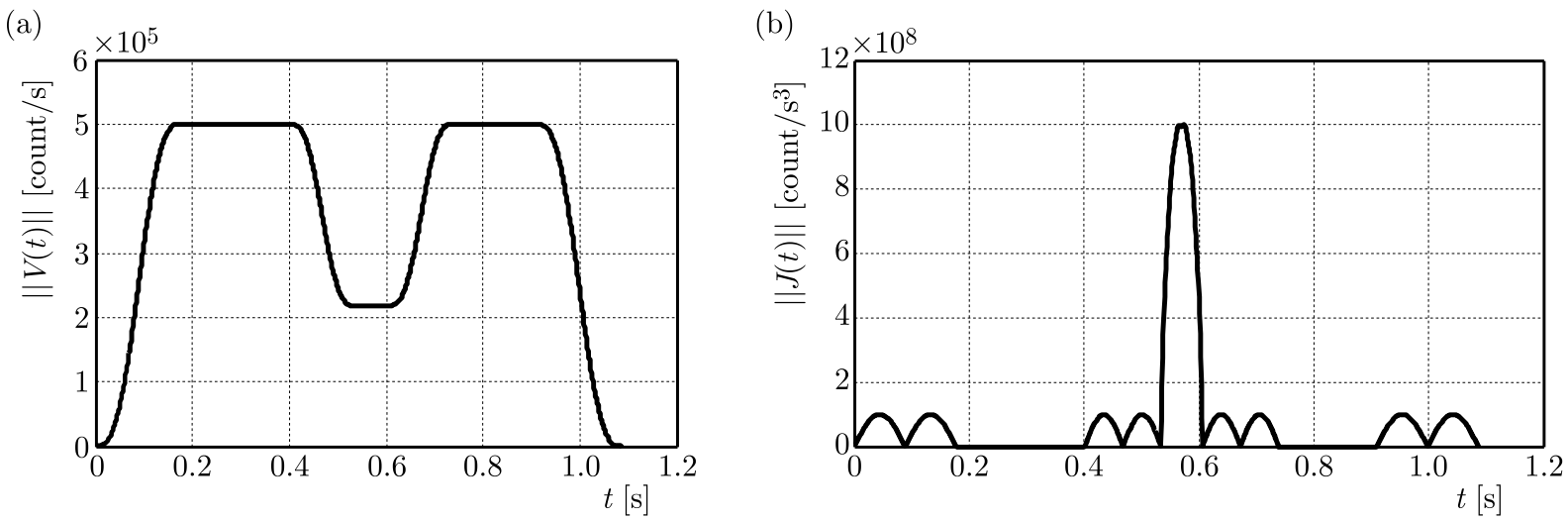

Fig. 1. (a) Velocity vector modulus. (b) Jerk vector modulus

In Fig. 1a, we can see a velocity decrease from $5 \cdot 10^{5}$ down to 218560.65 to satisfy the condition $\|\mathbf{J}(\sigma)\| \leqslant 10^{9}$. It happens on the transition curve between two lines constructed as a 3D septic polynomial. In Fig. 1b, we can see that the jerk comes exactly to the limiting value (time is measured in seconds, velocity in count $/ \mathrm{s}$, jerk in count $/ \mathrm{s}^{3}$ ). The calculations have been performed by (3.3) and (3.4). 


\section{References}

1. Angeles J., 2003, Fundamentals of Robotic Mechanical Systems: Theory, Methods and Algorithms, Springer

2. Farouki R.T., 2010, Pythagorean-Hodograph Curves, Springer 3

3. Sснот S.H., 1978, Jerk: the time rate of change of acceleration, American Journal of Physics, 46, 11, 1090-1105

Manuscript received December 1, 2016; accepted for print June 20, 2017 